\title{
Selective Inhibition of p38 MAPK Isoforms using Bipyrazole Derivatives: An in Silico Approach
}

\author{
N. Deepak \\ Assistant Professor \\ Department of CSE \\ Sir C R Reddy College of Engg \\ Eluru, Andhra Pradesh \\ India
}

\author{
Yesu Babu Adimulam, PhD \\ Professor and HoD \\ Department of CSE \\ Sir C R Reddy College of Engg \\ Eluru, Andhra Pradesh \\ India
}

\author{
R. Kiran Kumar, PhD \\ Assistant Professor \\ Department of Computer Science \\ Krishna University \\ Machilipatanam, Andhra Pradesh \\ India
}

\begin{abstract}
The p38 MAPK family of proteins comprised of four members; $338 \alpha, \mathrm{p} 38 \beta, \mathrm{p} 38 \gamma$, and p38 . All p38 MAPKs are strongly activated by environmental stresses and inflammatory cytokines. MAPKs have been shown to play a pivotal role in diverse diseases, including cancer. Majority of studies have focussed on targeting p38 MAPK isoform alpha. However, the report computational in silico molecular docking analysis of 34 bipyrazole analogs as possible p38 MAPK inhibitors. Results obtained are compared and it was observed that the top hit in all cases was found to be compound 26 with dock scores more than $200 \mathrm{kcal} / \mathrm{mol}$ whereas reported inhibitors in literature SB203580, SB202190, PD169316 resulted in dock scores between 164$189 \mathrm{kcal} / \mathrm{mol}$. Hence, it is worthwhile to study these bipyrazole analogs as a new class of p38 MAPK inhibitors.
\end{abstract}

\section{Keywords}

Docking, p38 MAPK Isoforms, Bipyrazoles

\section{INTRODUCTION}

The centre unit of mitogen-initiated protein kinase (MAPK) pathways is a three part protein kinase course. Inside of the three-kinase module, MAPKs are phosphorylated and enacted by MAPK kinases (MKKs). MKKs are typically double specificity kinases which catalyse the phosphorylation of MAPKs on both tyrosine and threonine build-ups. The MKKs are themselves phosphorylated and enacted by serine/threonine kinases; Xia et al. [1]

Initiation of MAPKs in light of these boosts controls quality expression, digestion system, cytoskeleton capacities and other cell administrative occasions. MAPKs add to complex administrative occasions including mitogenesis, separation, survival and movement; Schiller et al. [2]

The p38MAPK family contains four individuals, p38 $\alpha$, p38 $\beta$, p38 $\gamma$, and p386. All p38MAPKs are firmly initiated by ecological anxieties and provocative cytokines and are inadequately actuated by serum or development variables; Cuadrado et al. [3]. p38 is required for articulation of TNFa and interleukin-1 amid incendiary reactions. These kinases share profoundly comparative protein groupings; $\mathrm{p} 38 \alpha$ and $\mathrm{p} 38 \beta$ are $75 \%$ indistinguishable, though $\mathrm{p} 38 \gamma$ and $\mathrm{p} 38 \delta$ are $62 \%$ and $61 \%$ indistinguishable to $\mathrm{p} 38 \alpha$, individually. In addition, $\mathrm{p} 38 \gamma$ and $\mathrm{p} 38 \delta$ are $\sim 70 \%$ indistinguishable to one another. The four p38MAPK isoforms are broadly communicated; $\mathrm{p} 38 \beta$ is rich in cerebrum, p38 $\gamma$ in skeletal muscle, and $\mathrm{p} 38 \delta$ in endocrine organs; Cuenda et al.[4]. All isoforms are described by the vicinity of the preserved ThrGly-Tyr (TGY) phosphorylation theme in their initiation circle; Kumar et al.[5]. This theme is phosphorylated by MEK3 and MEK6, which themselves are initiated by different MAPKs that are incited by physical and compound burdens, for example, oxidative anxiety, hypoxia, X-beam and UV illumination and cytokines; Dhillon et al.[6]

In this paper, the contemplated in silico computational docking investigation of $\mathrm{p} 38$ MAPK isoforms with bipyrazole analogs and the coupling affinities are accounted for. The basis behind selecting bipyrazoles as could reasonably be expected p38 MAPK inhibitors depended on the earlier study [7].

\section{MATERIALS AND METHODS}

\subsection{Protein Targets}

The p38 MAPK protein isoforms used in the study are tabulated in Table 1. All targets are downloaded from PDB and subjected to molecular docking program Molegro. Before performing docking on a set of bipyrazole inhibitors, the docking protocol was validated by docking co-crystallized ligand with each protein. The results are given in Table 1 where the Root Mean Square Deviation of all inhibitors is less than $2.0 \mathrm{~A}^{\circ}$, indicating that the parameters for docking simulation are good in reproducing the geometric orientation of X-ray crystal structure.

Table 1: Co-crystallized Ligand Dock Scores of p38 MAPK Isoforms

\begin{tabular}{|l|l|l|l|l|}
\hline p38 MAPK isoform & PDB IDs & Description & $\begin{array}{l}\text { Dock Scores } \\
\text { (kcal/mol) }\end{array}$ & ${\text { RMSD }\left(\mathbf{A}^{\circ}\right)}$ \\
\hline p38-Alpha (MAPK14) & $1 \mathrm{~W} 82$ & $\begin{array}{l}\text { P38 Kinase Crystal Structure In Complex } \\
\text { With Small Molecule Inhibitor }\end{array}$ & -164.37 & 0.554 \\
\hline p38-Beta (MAPK11) & $3 \mathrm{GP0}$ & $\begin{array}{l}\text { Crystal Structure of Human Mitogen } \\
\text { Activated Protein Kinase 11 (p38 beta) in } \\
\text { complex with Nilotinib }\end{array}$ & -191.44 & 1.945 \\
\hline p38-Gamma (MAPK12) & 1CM8 & Phosphorylated Map Kinase P38-Gamma & -155.46 & 1.649 \\
\hline p38-Delta (MAPK13) & 4EYJ & MAPK13 Complex with inhibitor & -149.47 & 1.066 \\
\hline
\end{tabular}




\subsection{Bipyrazole Derivatives}

In the earlier report[7], the detailed lab studied inhibitory activities of compounds containing urea, pyrazole, bipyrazole, thiazole, thiadiazole etc scaffold analogs against p38-alpha MAPK, where it was observed that better binding affinities were reported by bipyrazole analogs than the remaining.
Hence, the extension of this study to enumerate the specificity of binding of bipyrazoles [9-10] towards p38-alpha, p-38beta, p38-gamma and p-38-delta proteins which participate in Inflammatory cytokine pathway. The IUPAC names and SMILES string of all 34 bipyrazoles are given in Table-2.

Table 2: IUPAC Names and SMILES String of all 34 Bipyrazoles

\begin{tabular}{|c|c|c|}
\hline $\begin{array}{l}\text { Compound } \\
\text { ID }\end{array}$ & IUPAC Name & SMILES \\
\hline 1 & $\begin{array}{l}\text { ethyl 5-amino-3-anilino-1H-pyrazole-4- } \\
\text { carboxylate }\end{array}$ & $\operatorname{CCOC}(=\mathrm{O}) \mathrm{c} 1 \mathrm{c}(\mathrm{N})[\mathrm{nH}] \mathrm{nc} 1 \mathrm{Nc} 2 \mathrm{ccccc} 2$ \\
\hline 2 & $\begin{array}{l}\text { ethyl 5-amino-1-(5-amino-3-anilino-4- } \\
\text { ethoxycarbonyl-pyrazol-1-yl)-3-anilino- } \\
\text { pyrazole-4-carboxylate }\end{array}$ & $\begin{array}{l}\operatorname{CCOC}(=\mathrm{O}) \mathrm{c} 1 \mathrm{c}(\mathrm{N}) \mathrm{n}(\mathrm{nc} 1 \mathrm{Nc} 2 \mathrm{ccccc} 2) \mathrm{n} 3 \mathrm{nc} \\
(\mathrm{Nc} 4 \mathrm{ccccc} 4) \mathrm{c}(\mathrm{C}(=\mathrm{O}) \mathrm{OCC}) \mathrm{c} 3 \mathrm{~N}\end{array}$ \\
\hline 3 & $\begin{array}{l}\text { ethyl 5-amino-1-(4-chloro-4- } \\
\text { ethoxycarbonyl-5-oxo-1H-pyrazol-3-yl)- } \\
\text { 3-ethoxy-pyrazole-4-carboxylate }\end{array}$ & $\begin{array}{l}\mathrm{CCOC}(=\mathrm{O}) \mathrm{c} 1 \mathrm{c}(\mathrm{N}) \mathrm{n}(\mathrm{nc} 1 \mathrm{OCC}) \mathrm{C} 2=\mathrm{NNC}(=\mathrm{O}) \\
\mathrm{C} 2(\mathrm{Cl}) \mathrm{C}(=\mathrm{O}) \mathrm{OCC}\end{array}$ \\
\hline 4 & $\begin{array}{l}\text { 5-(4-chlorophenyl)-4-(4-cyanopyrazol-1- } \\
\text { yl)-N-(4-phenylphenyl)-3,4- } \\
\text { dihydropyrazole-2-carboxamide }\end{array}$ & $\begin{array}{l}\mathrm{Clc} 1 \mathrm{ccc}(\mathrm{cc} 1) \mathrm{C} 2=\mathrm{NN}(\mathrm{CC} 2 \mathrm{n} 3 \mathrm{cc}(\mathrm{cn} 3) \mathrm{C \# N}) \mathrm{C}(=\mathrm{O}) \\
\mathrm{Nc} 4 \operatorname{ccc}(\mathrm{cc} 4) \mathrm{c} 5 \mathrm{ccccc} 5\end{array}$ \\
\hline 5 & $\begin{array}{l}\text { 1-(1,5-diphenylpyrazol-4-yl)-3,5- } \\
\text { dimethyl-pyrazole }\end{array}$ & Cc1cc(C)n(n1)c2enn(c3eccec3)c2c4cccec4 \\
\hline 6 & $\begin{array}{l}\text { methyl 4-(3,5-dimethylpyrazol-1-yl)-5- } \\
\text { phenyl-pyrazole-1-carboxylate }\end{array}$ & $\operatorname{COC}(=\mathrm{O}) \mathrm{n} 1 \mathrm{ncc}(\mathrm{c} 1 \mathrm{c} 2 \mathrm{cccc} 2) \mathrm{n} 3 \mathrm{nc}(\mathrm{C}) \mathrm{cc} 3 \mathrm{C}$ \\
\hline 7 & $\begin{array}{l}\text { 1-tert-butyl-4-(3,5-dimethylpyrazol-1- } \\
\text { yl)-5-phenyl-pyrazole }\end{array}$ & $\mathrm{Cc} 1 \mathrm{cc}(\mathrm{C}) \mathrm{n}(\mathrm{n} 1) \mathrm{c} 2 \mathrm{cnn}(\mathrm{c} 2 \mathrm{c} 3 \operatorname{ccc} c 3) \mathrm{C}(\mathrm{C})(\mathrm{C}) \mathrm{C}$ \\
\hline 8 & $\begin{array}{l}\text { bis(2-adamantyl)-[2-[1-(4- } \\
\text { methoxyphenyl)-3,5-diphenyl-pyrazol-4- } \\
\text { yl]pyrazol-3-yl]phosphane }\end{array}$ & $\begin{array}{l}\mathrm{COc} 1 \mathrm{ccc}(\mathrm{cc} 1) \mathrm{n} 2 \mathrm{nc}(\mathrm{c} 3 \mathrm{cccc} 3) \mathrm{c}(\mathrm{c} 2 \mathrm{c} 4 \mathrm{cccc} 4) \mathrm{n} 5 \mathrm{nccc} \\
5 \mathrm{P}(\mathrm{C} 6 \mathrm{C} 7 \mathrm{CC} 8 \mathrm{CC}(\mathrm{CC} 6 \mathrm{C} 8) \mathrm{C} 7) \mathrm{C} 9 \mathrm{C} \% 10 \mathrm{CC} \% 11 \mathrm{CC}(\mathrm{CC} 9 \mathrm{C} \% 11) \\
\mathrm{C} \% 10\end{array}$ \\
\hline 9 & $\begin{array}{l}\text { dicyclohexyl-[2-[1-(4-methoxyphenyl)- } \\
\text { 3,5-diphenyl-pyrazol-4-yl]pyrazol-3- } \\
\text { yl]phosphane }\end{array}$ & $\begin{array}{l}\text { COc1ccc(cc1)n2nc(c3cccc3)c(c2c4ccccc4)n5nccc5P } \\
(\text { C6CCCCC6)C7CCCCC7 }\end{array}$ \\
\hline 10 & $\begin{array}{l}\text { ditert-butyl-[2-[1-(4-methoxyphenyl)- } \\
\text { 3,5-diphenyl-pyrazol-4-yl]pyrazol-3- } \\
\text { yl]phosphane }\end{array}$ & $\begin{array}{l}\text { COc1ccc }(\mathrm{cc} 1) n 2 \mathrm{nc}(\mathrm{c} 3 \mathrm{ccccc} 3) \mathrm{c}(\mathrm{c} 2 \mathrm{c} 4 \mathrm{ccccc} 4) \mathrm{n} 5 \mathrm{nccc} 5 \mathrm{P}(\mathrm{C} \\
(\mathrm{C})(\mathrm{C}) \mathrm{C}) \mathrm{C}(\mathrm{C})(\mathrm{C}) \mathrm{C}\end{array}$ \\
\hline 11 & $\begin{array}{l}\text { 4-chloro-1-(3,5-dinitro-1H-pyrazol-4-yl)- } \\
\text { 5-nitro-pyrazole }\end{array}$ & {$[\mathrm{O}-][\mathrm{N}+](=\mathrm{O}) \mathrm{c} 1 \mathrm{n}[\mathrm{nH}] \mathrm{c}(\mathrm{c} 1 \mathrm{n} 2 \mathrm{ncc}(\mathrm{Cl}) \mathrm{c} 2[\mathrm{~N}+](=\mathrm{O})[\mathrm{O}-])[\mathrm{N}+](=\mathrm{O})[\mathrm{O}-]$} \\
\hline 12 & $\begin{array}{l}\text { 1-(3,5-dinitro-1H-pyrazol-4-yl)-4,5- } \\
\text { dinitro-pyrazole }\end{array}$ & $\begin{array}{l}{[\mathrm{O}-][\mathrm{N}+](=\mathrm{O}) \mathrm{c} 1 \mathrm{cnn}(\mathrm{c} 1[\mathrm{~N}+](=\mathrm{O})[\mathrm{O}-]) \mathrm{c} 2 \mathrm{c}(\mathrm{n}[\mathrm{nH}] \mathrm{c} 2[\mathrm{~N}+](=\mathrm{O})[\mathrm{O}-} \\
])[\mathrm{N}+](=\mathrm{O})[\mathrm{O}-]\end{array}$ \\
\hline 13 & $\begin{array}{l}\text { 1-methyl-3,4-dinitro-5-(3-nitropyrazol-1- } \\
\text { yl)pyrazole }\end{array}$ & $\mathrm{Cn} 1 \mathrm{nc}(\mathrm{c}(\mathrm{c} \ln 2 \operatorname{ccc}(\mathrm{n} 2)[\mathrm{N}+](=\mathrm{O})[\mathrm{O}-])[\mathrm{N}+](=\mathrm{O})[\mathrm{O}-])[\mathrm{N}+](=\mathrm{O})[\mathrm{O}-]$ \\
\hline 14 & $\begin{array}{l}\text { 1-methyl-3,4-dinitro-5-(4-nitropyrazol-1- } \\
\text { yl)pyrazole }\end{array}$ & $\mathrm{Cn} 1 \mathrm{nc}(\mathrm{c}(\mathrm{c} \ln 2 \mathrm{cc}(\mathrm{cn} 2)[\mathrm{N}+](=\mathrm{O})[\mathrm{O}-])[\mathrm{N}+](=\mathrm{O})[\mathrm{O}-])[\mathrm{N}+](=\mathrm{O})[\mathrm{O}-]$ \\
\hline 15 & $\begin{array}{l}\mathrm{N} \text {-[1-(4-methoxyphenyl)-3-methyl-5- } \\
\text { pyrazol-1-yl-pyrazol-4- } \\
\text { yl]methanesulfonamide }\end{array}$ & $\mathrm{COc} 1 \mathrm{ccc}(\mathrm{cc} 1) \mathrm{n} 2 \mathrm{nc}(\mathrm{C}) \mathrm{c}(\mathrm{NS}(=\mathrm{O})(=\mathrm{O}) \mathrm{C}) \mathrm{c} 2 \mathrm{n} 3 \operatorname{ccc} \mathrm{3}$ \\
\hline 16 & $\begin{array}{l}\mathrm{N} \text {-[1-(4-bromophenyl)-3-methyl-5- } \\
\text { pyrazol-1-yl-pyrazol-4- } \\
\text { yl]methanesulfonamide }\end{array}$ & $\mathrm{Cc} 1 \mathrm{nn}(\mathrm{c} 2 \mathrm{ccc}(\mathrm{Br}) \mathrm{cc} 2) \mathrm{c}(\mathrm{c} 1 \mathrm{NS}(=\mathrm{O})(=\mathrm{O}) \mathrm{C}) \mathrm{n} 3 \mathrm{ccc} 3$ \\
\hline 17 & $\begin{array}{l}\mathrm{N} \text {-[1-(4-chlorophenyl)-3-methyl-5- } \\
\text { pyrazol-1-yl-pyrazol-4- }\end{array}$ & $\mathrm{Cc} 1 \mathrm{nn}(\mathrm{c} 2 \mathrm{ccc}(\mathrm{Cl}) \mathrm{cc} 2) \mathrm{c}(\mathrm{c} 1 \mathrm{NS}(=\mathrm{O})(=\mathrm{O}) \mathrm{C}) \mathrm{n} 3 \mathrm{cccn} 3$ \\
\hline
\end{tabular}




\begin{tabular}{|c|c|c|}
\hline & yl]methanesulfonamide & \\
\hline 18 & $\begin{array}{l}\mathrm{N} \text {-[1-(4-fluorophenyl)-3-methyl-5- } \\
\text { pyrazol-1-yl-pyrazol-4- } \\
\text { yl]methanesulfonamide }\end{array}$ & $\mathrm{Cc} 1 \mathrm{nn}(\mathrm{c} 2 \mathrm{ccc}(\mathrm{F}) \mathrm{cc} 2) \mathrm{c}(\mathrm{c} 1 \mathrm{NS}(=\mathrm{O})(=\mathrm{O}) \mathrm{C}) \mathrm{n} 3 \mathrm{ccc} 3$ \\
\hline 19 & $\begin{array}{l}\mathrm{N} \text {-[3-methyl-1-(4-nitrophenyl)-5- } \\
\text { pyrazol-1-yl-pyrazol-4- } \\
\text { yl]methanesulfonamide }\end{array}$ & $\begin{array}{l}\mathrm{Cc} 1 \mathrm{nn}(\mathrm{c} 2 \operatorname{ccc}(\operatorname{cc} 2)[\mathrm{N}+](=\mathrm{O})[\mathrm{O}-]) \mathrm{c}(\operatorname{coNS}(=\mathrm{O})(=\mathrm{O}) \mathrm{C}) \\
\mathrm{n} 3 \operatorname{ccc} 3\end{array}$ \\
\hline 20 & $\begin{array}{l}\text { ethyl 5-amino-1-(5-methyl-4-nitro-2- } \\
\text { phenyl-pyrazol-3-yl)pyrazole-4- } \\
\text { carboxylate }\end{array}$ & $\begin{array}{l}\operatorname{CCOC}(=\mathrm{O}) \mathrm{c} 1 \operatorname{cnn}(\mathrm{c} 1 \mathrm{~N}) \mathrm{c} 2 \mathrm{c}(\mathrm{c}(\mathrm{C}) \mathrm{nn} 2 \mathrm{c} 3 \operatorname{ccccc} 3)[\mathrm{N}+] \\
(=\mathrm{O})[\mathrm{O}-]\end{array}$ \\
\hline 21 & $\begin{array}{l}\text { 3-acetyl-1-(4-bromo-3-phenyl-1H- } \\
\text { pyrazol-5-yl)-5-phenyl-pyrazole-4- } \\
\text { carbonitrile }\end{array}$ & $\begin{array}{l}\mathrm{CC}(=\mathrm{O}) \mathrm{c} 1 \mathrm{nn}(\mathrm{c}(\mathrm{c} 2 \mathrm{ccccc} 2) \mathrm{c} 1 \mathrm{C \# N}) \mathrm{c} 3[\mathrm{nH}] \mathrm{nc}(\mathrm{c} 3 \mathrm{Br}) \\
\mathrm{c} 4 \mathrm{ccccc} 4\end{array}$ \\
\hline 22 & $\begin{array}{l}\text { ethyl 3-acetyl-5-amino-1-(4-bromo-3- } \\
\text { phenyl-1H-pyrazol-5-yl)pyrazole-4- } \\
\text { carboxylate }\end{array}$ & $\begin{array}{l}\operatorname{CCOC}(=\mathrm{O}) \mathrm{c} 1 \mathrm{c}(\mathrm{N}) \mathrm{n}(\mathrm{nc} 1 \mathrm{C}(=\mathrm{O}) \mathrm{C}) \mathrm{c} 2[\mathrm{nH}] \mathrm{nc}(\mathrm{c} 2 \mathrm{Br}) \\
\mathrm{c} 3 \operatorname{ccccc} 3\end{array}$ \\
\hline 23 & $\begin{array}{l}\text { 1-(4-nitrophenyl)-3-[1-(4-nitrophenyl)-5- } \\
\text { propyl-pyrazol-3-yl]-5-propyl-pyrazole }\end{array}$ & $\begin{array}{l}\text { CCCc1cc(nn1c2 } \operatorname{ccc}(\operatorname{cc} 2)[\mathrm{N}+](=\mathrm{O})[\mathrm{O}-]) \mathrm{c} 3 \operatorname{cc}(\mathrm{CCC}) \mathrm{n} \\
(\mathrm{n} 3) \mathrm{c} 4 \operatorname{ccc}(\mathrm{cc} 4)[\mathrm{N}+](=\mathrm{O})[\mathrm{O}-]\end{array}$ \\
\hline 24 & $\begin{array}{l}\text { 5-isopropyl-3-[5-isopropyl-1-(4- } \\
\text { nitrophenyl)pyrazol-3-yl]-1-(4- } \\
\text { nitrophenyl)pyrazole }\end{array}$ & $\begin{array}{l}\mathrm{CC}(\mathrm{C}) \mathrm{c} 1 \mathrm{cc}(\mathrm{nn} 1 \mathrm{c} 2 \operatorname{ccc}(\mathrm{cc} 2)[\mathrm{N}+](=\mathrm{O})[\mathrm{O}- \\
]) \operatorname{coc}(\mathrm{C}(\mathrm{C}) \mathrm{C}) \mathrm{n}(\mathrm{n} 3) \operatorname{c} 4 \operatorname{ccc}(\mathrm{cc} 4)[\mathrm{N}+](=\mathrm{O})[\mathrm{O}-]\end{array}$ \\
\hline 25 & $\begin{array}{l}\text { 5-[5-carbamoyl-1-(2,4-dichlorophenyl)- } \\
\text { 4H-pyrazol-3-yl]-2-(2,4- } \\
\text { dichlorophenyl)pyrazole-3-carboxamide }\end{array}$ & $\begin{array}{l}\mathrm{NC}(=\mathrm{O}) \mathrm{C} 1=[\mathrm{N}](\mathrm{N}=\mathrm{C}(\mathrm{C} 1) \mathrm{c} 2 \mathrm{cc}(\mathrm{C}(=\mathrm{O}) \mathrm{N}) \mathrm{n}(\mathrm{n} 2) \mathrm{c} 3 \mathrm{ccc}(\mathrm{Cl}) \mathrm{cc} 3 \mathrm{Cl}) \\
\mathrm{c} 4 \mathrm{ccc}(\mathrm{Cl}) \mathrm{cc} 4 \mathrm{Cl}\end{array}$ \\
\hline 26 & $\begin{array}{l}\text { 2-[5-[5-(1,3-benzothiazol-2-yl)-1,4-bis(4- } \\
\text { chlorophenyl)pyrazol-3-yl]-2,4-bis(4- } \\
\text { chlorophenyl)-4H-pyrazol-3-yl]-1,3- } \\
\text { benzothiazole }\end{array}$ & $\begin{array}{l}\mathrm{Clc} 1 \mathrm{ccc}(\mathrm{cc} 1) \mathrm{C} 2 \mathrm{C}(=\mathrm{N}[\mathrm{N}](=\mathrm{C} 2 \mathrm{c} 3 \mathrm{nc} 4 \mathrm{cccc} 4 \mathrm{~s} 3) \mathrm{c} 5 \operatorname{ccc}(\mathrm{Cl}) \mathrm{cc} 5) \\
\mathrm{c} 6 \mathrm{nn}(\mathrm{c} 7 \mathrm{ccc}(\mathrm{Cl}) \mathrm{cc} 7) \mathrm{c}(\mathrm{c} 8 \mathrm{nc} 9 \mathrm{ccccc} 9 \mathrm{~s} 8) \mathrm{c} 6 \mathrm{c} \% 10 \mathrm{ccc}(\mathrm{Cl}) \mathrm{cc} \% 10\end{array}$ \\
\hline 27 & $\begin{array}{l}\text { [2-(4-chlorophenyl)-5-[1-(4- } \\
\text { chlorophenyl)-5-(2-hydroxybenzoyl)-4- } \\
\text { phenyl-4H-pyrazol-3-yl]-4-phenyl- } \\
\text { pyrazol-3-yl]-(2- } \\
\text { hydroxyphenyl)methanone }\end{array}$ & $\begin{array}{l}\mathrm{Oc} 1 \operatorname{cccc} 1 \mathrm{C}(=\mathrm{O}) \mathrm{C} 2=[\mathrm{N}](\mathrm{N}=\mathrm{C}(\mathrm{C} 2 \mathrm{c} 3 \operatorname{cccc} 3) \mathrm{c} 4 \mathrm{nn}(\mathrm{c} 5 \operatorname{ccc}(\mathrm{Cl}) \\
\operatorname{cc} 5) \mathrm{c}(\mathrm{C}(=\mathrm{O}) \operatorname{c} 6 \operatorname{ccc} c 6 \mathrm{O}) \mathrm{c} 4 \mathrm{c} 7 \mathrm{cccc} 7) \mathrm{c} 8 \mathrm{ccc}(\mathrm{Cl}) \mathrm{cc} 8\end{array}$ \\
\hline 28 & $\begin{array}{l}\text { 1-(4-chlorophenyl)-5-phenyl-3-(1H- } \\
\text { pyrazol-3-yl)pyrazole-4-carbohydrazide }\end{array}$ & $\mathrm{NNC}(=\mathrm{O}) \mathrm{c} 1 \mathrm{c}(\mathrm{nn}(\mathrm{c} 2 \mathrm{ccc}(\mathrm{Cl}) \mathrm{cc} 2) \mathrm{c} 1 \mathrm{c} 3 \mathrm{ccccc} 3) \mathrm{c} 4 \mathrm{cc}[\mathrm{nH}] \mathrm{n} 4$ \\
\hline 29 & $\begin{array}{l}\text { 4-[(4Z)-5-amino-4-[(4- } \\
\text { bromophenyl)methylene]pyrazol-3-yl]- } \\
\text { 1,5-dimethyl-2-phenyl-pyrazol-3-one }\end{array}$ & $\begin{array}{l}\mathrm{CN} 1 \mathrm{~N}(\mathrm{C}(=\mathrm{O}) \mathrm{C}(=\mathrm{C} 1 \mathrm{C}) \mathrm{C} 2=\mathrm{NN}=\mathrm{C}(\mathrm{N}) / \mathrm{C} / 2=\mathrm{Clc} 3 \operatorname{ccc}(\mathrm{Br}) \mathrm{cc} 3) \\
\mathrm{c} 4 \operatorname{ccccc} 4\end{array}$ \\
\hline 30 & $\begin{array}{l}\text { (E)-3-(2-hydroxyphenyl)-1-[1-phenyl-3- } \\
\text { (2-thienyl)pyrazol-4-yl]prop-2-en-1-one }\end{array}$ & 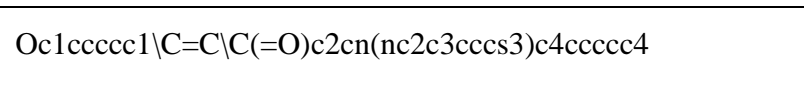 \\
\hline 31 & $\begin{array}{l}\text { 5-methyl-4-[5-(4-oxochromen-3-yl)-4,5- } \\
\text { dihydro-1H-pyrazol-3-yl]-1,2- } \\
\text { dihydropyrazol-3-one }\end{array}$ & $\mathrm{CC} 1=\mathrm{C}(\mathrm{C}(=\mathrm{O}) \mathrm{NN} 1) \mathrm{C} 2=\mathrm{NNC}(\mathrm{C} 2) \mathrm{C} 3=\mathrm{CO} 4 \operatorname{ccccc} 4 \mathrm{C} 3=\mathrm{O}$ \\
\hline 32 & $\begin{array}{l}\text { 5-amino-N-(1,3-benzothiazol-2-yl)-3- } \\
\text { (1,3-diphenylpyrazol-4-yl)-1H-pyrazole- } \\
\text { 4-carboxamide }\end{array}$ & $\begin{array}{l}\mathrm{Nc} 1[\mathrm{nH}] \mathrm{nc}(\mathrm{c} 2 \mathrm{cn}(\mathrm{nc} 2 \mathrm{c} 3 \mathrm{ccccc} 3) \mathrm{c} 4 \mathrm{ccccc} 4) \mathrm{c} 1 \mathrm{C}(=\mathrm{O}) \mathrm{N} \\
\mathrm{c} 5 \mathrm{nc} 6 \mathrm{ccccc} 6 \mathrm{~s} 5\end{array}$ \\
\hline 33 & $\begin{array}{l}\text { 3-(5-hydroxy-3-methyl-1-phenyl- } \\
\text { pyrazol-4-yl)-1H-pyrazole-5- } \\
\text { carbohydrazide }\end{array}$ & $\mathrm{Cc} 1 \mathrm{nn}(\mathrm{c}(\mathrm{O}) \mathrm{c} 1 \mathrm{c} 2 \mathrm{cc}([\mathrm{nH}] \mathrm{n} 2) \mathrm{C}(=\mathrm{O}) \mathrm{NN}) \mathrm{c} 3 \operatorname{ccccc} 3$ \\
\hline 34 & $\begin{array}{l}\text { diethyl 2-(4-bromophenyl)-5-(4-cyano-5- } \\
\text { methyl-2-phenyl-pyrazol-3-yl)pyrazole- } \\
\text { 3,4-dicarboxylate }\end{array}$ & $\begin{array}{l}\mathrm{CCOC}(=\mathrm{O}) \mathrm{c} 1 \mathrm{c}(\mathrm{nn}(\mathrm{c} 2 \mathrm{ccc}(\mathrm{Br}) \mathrm{cc} 2) \mathrm{c} 1 \mathrm{C}(=\mathrm{O}) \mathrm{OCC}) \mathrm{c} 3 \mathrm{c}(\mathrm{C \# N}) \\
\mathrm{c}(\mathrm{C}) \mathrm{nn} 3 \mathrm{c} 4 \mathrm{ccccc} 4\end{array}$ \\
\hline
\end{tabular}




\subsection{Molecular Docking}

To gain better insight of the interactions between various compounds and p38 alpha MAPK, Molegro Virtual Docker was employed to dock the selected experimentally non-tested inhibitors from literature. Molegro requires the receptor and ligand coordinates in either Mol2 or PDB format. Nonpolar hydrogen atoms were removed from the receptor file and bonds, bond orders, hybridization; partial charges were added to the corresponding atoms. Expanded molecular surface cavities were detected and the cavity representing ligand was selected as binding site during docking runs. Mol Dock algorithm with default parameters such as 1500 iterations with maximum 50 as population size, 300 steps and 10 runs were employed.

\section{RESULTS AND DISCUSSION}

When compared with p38 isoforms, majority of the studies have reported on p38-alpha MAPK, owing to the fact that p38alpha activation has been observed in many hematopoietic and non-hematopoietic cell types upon appropriate stimuli. Following the discovery of pyridinyl-imidazole- inhibitor, progress of research and efforts have been placed by many companies to develop p38alpha inhibitors as potential treatments for inflammatory diseases; Dominguez et al.[11].

\subsection{P38alpha (MAPK14)}

P38alpha MAPK protein 1W82 bound ligand L10 resulted in a dock score of $-164.37 \mathrm{kcal} / \mathrm{mol}$ showed two major H-bond interactions with Glu71 and Asp168 residues, respectively. Docking simulations of 34 bipyrazoles resulted in high binding affinity of $-242.002 \mathrm{kcal} / \mathrm{mol}$ exhibited by compound 26 and displayed three H-bond interactions (Arg67, Asp168 and Arg149) with active site residues of $1 \mathrm{~W} 82$. The top 3 compounds are given in Table 3 , which enumerates the role of bipyrazoles as possible p38 alpha MAPK inhibitors.

Table 3. 2-D Structure Images and Docking Scores of Top three Bipyrazole Derivatives Exhibited High Binding Affinity against $1 \mathrm{W82}$

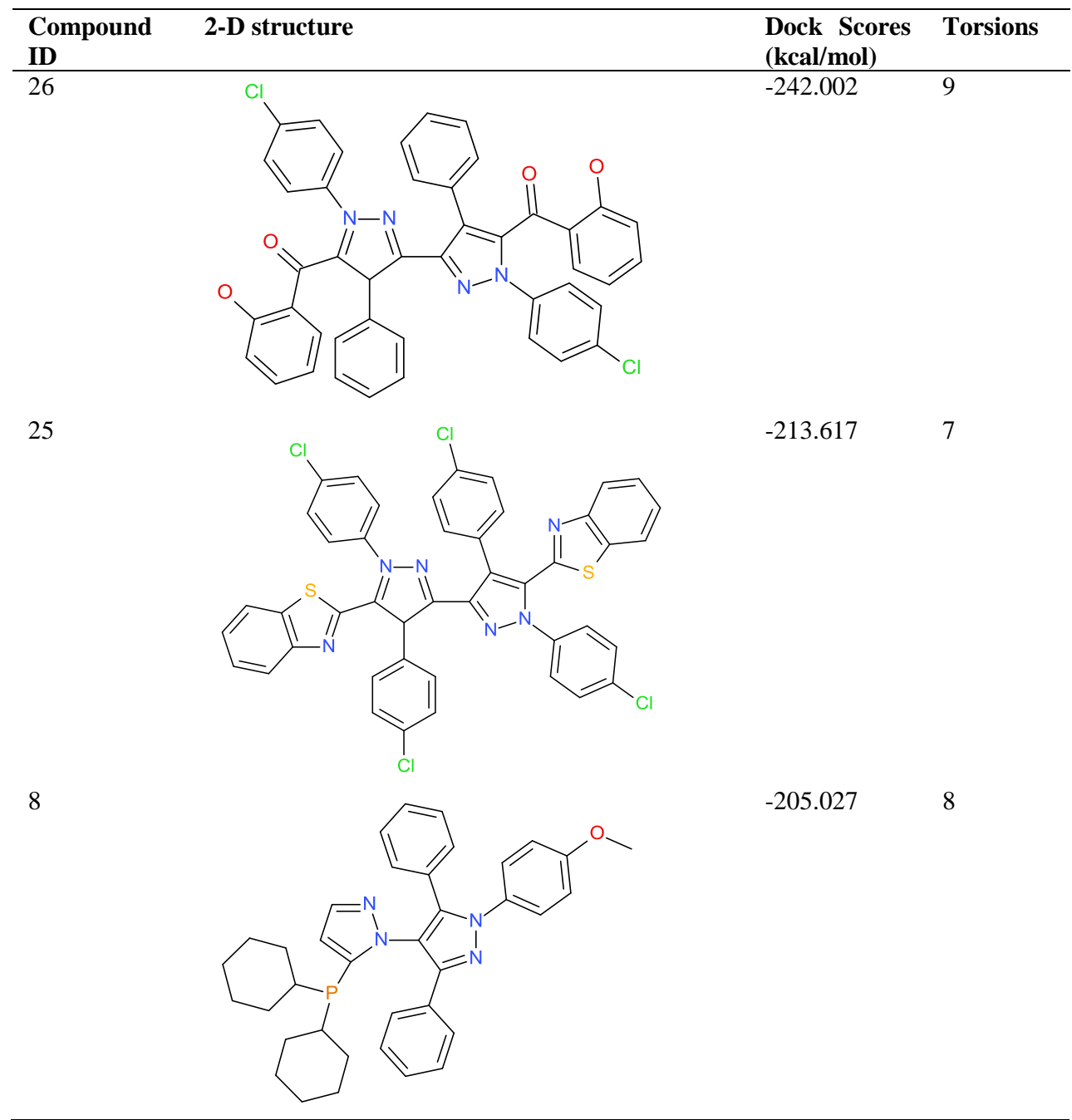

\subsection{P38beta (MAPK11)}

The p38 MAPK alpha and beta isoforms are expressed throughout the body and are found in many different cell types. These two isoforms are inhibited by certain known small molecule p38 MAPK inhibitors. For example, SB203580 is a specific inhibitor of p38 alpha and beta which suppresses downstream activation of MAPKAP kinase-2 and heat shock protein. Docking bipyrazoles with MAPK11 resulted in dock score of $-231.953 \mathrm{kcal} / \mathrm{mol}$ by compound 26 , which is much higher than co-crystallized ligand of $3 \mathrm{GP} 0$, $191.44 \mathrm{kcal} / \mathrm{mol}$. Interestingly, the top 3 compounds in p38 MAPk alpha are similar in case of p38 beta and are shown to 
exhibit dual inhibitory properties against p38 MAPK alpha and beta. The effectiveness of similar ligands in both the cases suggests the fact that the top compound 26 fits exactly within the geometric active site regions of p38 alpha and beta and hence better binding affinities. The results are given in Table 4.

Table 4. 2-D structure images and docking scores of top three bipyrazole derivatives exhibited high binding affinity against 3GPO

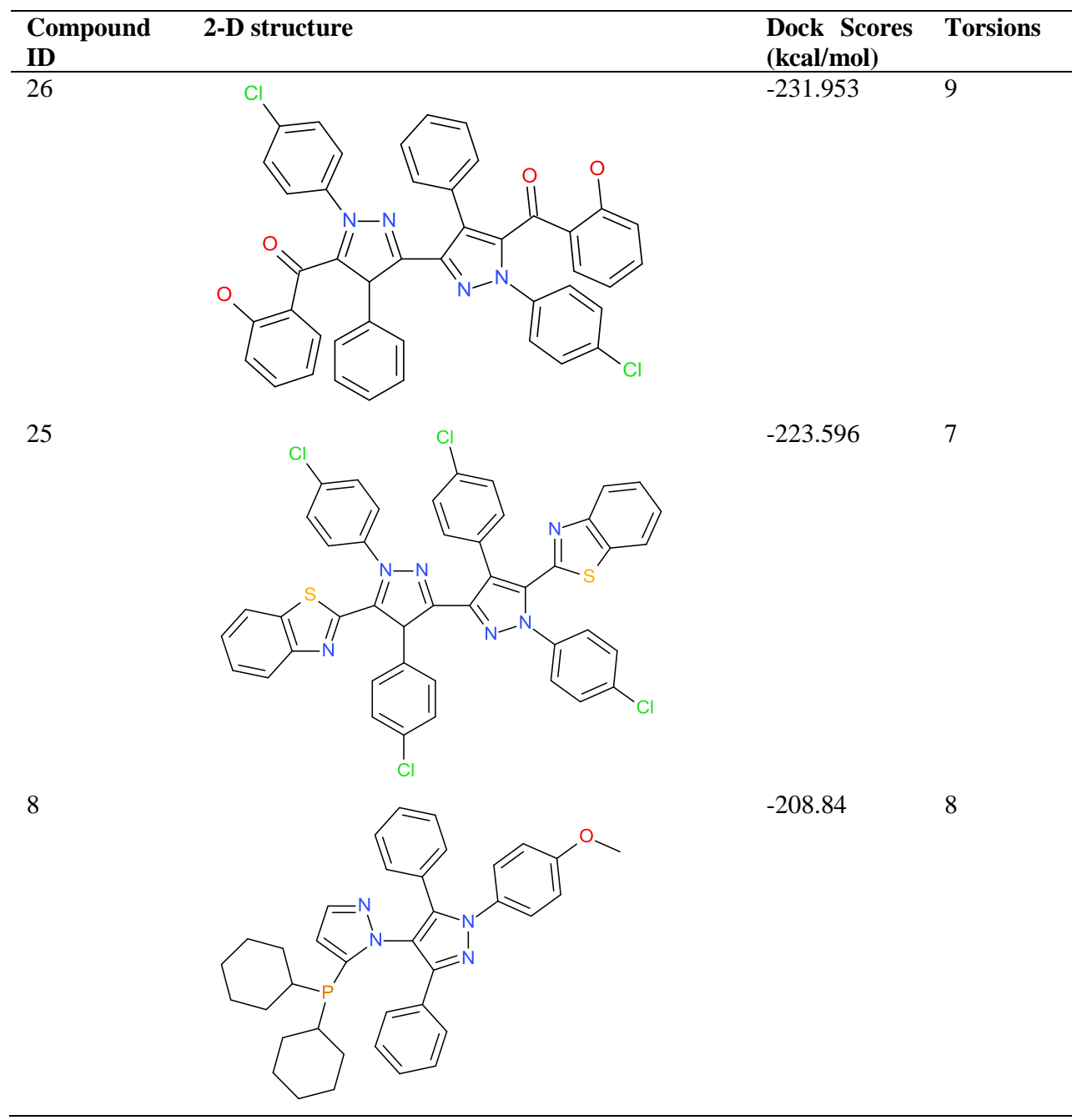

\subsection{P38 gamma (MAPK12)}

In this case, the top three hits are reported by compounds 26 , 25 and 9 , respectively, with best dock score $-211.751 \mathrm{kcal} / \mathrm{mol}$ displayed by compound 26 when docked with 1CM8 showing
Gly157 and Lys118 H-bond interactions. The co-crystallized ligand of $1 \mathrm{CM} 8$ reported a score of $-155.46 \mathrm{kcal} / \mathrm{mol}$. The data is given in table 5 .

Table 5. 2-D structure images and docking scores of top three bipyrazole derivatives exhibited high binding affinity against 3GP0

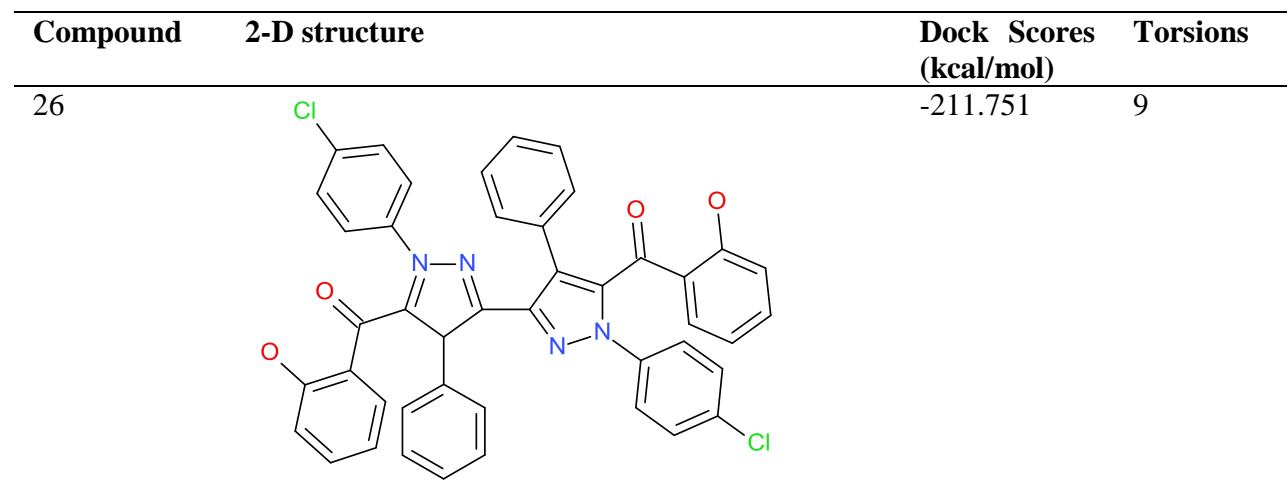


25<smiles></smiles>

9<smiles>COc1ccc(-n2nc(-c3ccccc3)c(-c3ccccc3)c2-n2ccnc2P(C(C)(C)C)C(C)(C)C)cc1</smiles>

$-202.149$

7

$-175.289 \quad 7$

\subsection{P38 delta (MAPK13)}

The top three hits obtained in this case are little different from the remaining, however, the best dock score -232.834 $\mathrm{kcal} / \mathrm{mol}$ was displayed by compound 26 when docked with
4EYJ showing H-bond interactions with His148 and Asp168. The co-crystallized ligand of 4EYJ reported a score of -155.46 $\mathrm{kcal} / \mathrm{mol}$. The data is given in table 6 .

Table 6. 2-D structure images and docking scores of top three bipyrazole derivatives exhibited high binding affinity against 4EYJ

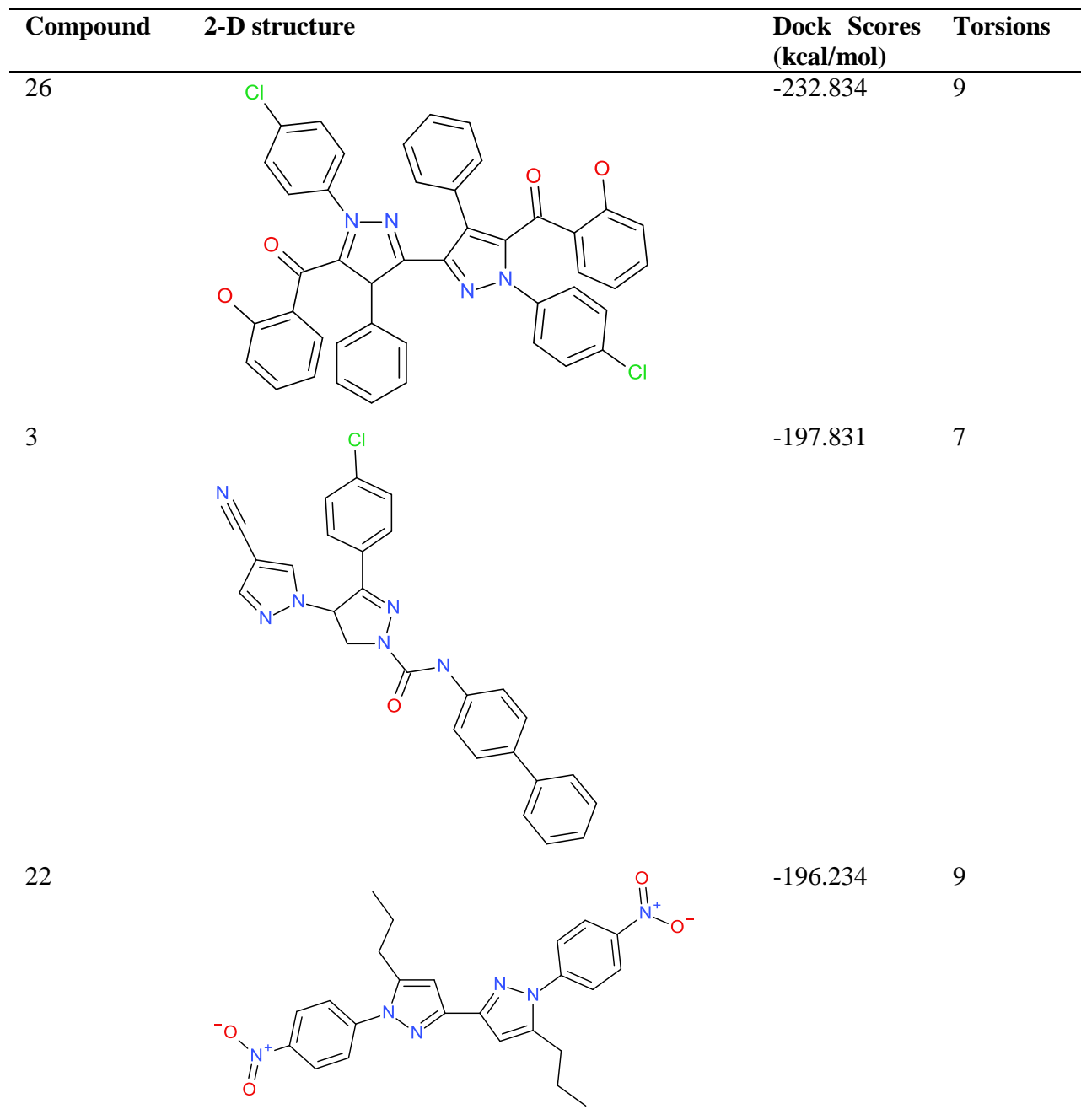


From the above data, it is clearly evident that compound 26 was found to interact effectively than the remaining compounds as well as co-crystallized ligands of all p38 MAPK isoforms. This in silico investigation suggested the importance of bipyrazole analogs as possible inhibitors of p38 MAPKs. Considering the fact that solubility of a compound depends partially on the molecular weights of the compound, it can be emphasized that the next best compounds have mass less than or equal to 500, a feature important in Lipinski's rule of 5. Moreover, the top three compounds in each case have effective binding energies than bound ligands of all p38 MAPK proteins. Compound 26 looks like a steroisomeric structure when cleaved into two halves which proposes that the orientation of this molecule would have an overall effect on the interaction features with active site residues of $\mathrm{p} 38$ MAPK. All isoforms exhibited compound 26 as best inhibitor and hence it is hereby suggested that bipyrazole groups might be studied for p38 MAPK inhibition.

From literature, it was found that few p38 MAPK inhibitors such as SB203580, SB202190, and PD169316 have been reported and hence these compounds when docked against four isoforms of p38 MAPK resulted in dock scores between $164-189 \mathrm{kcal} / \mathrm{mol}$.

\section{CONCLUSIONS}

Of all the $\mathrm{p} 38$ MAPK isoforms, $\alpha$ and $\beta$ have been reported as essential targets in various pathological processes and hence few inhibitors are reported in literature. This study focussed on studying reported inhibitors such as SB203580, SB202190, PD169316 as well as novel bipyrazole derivatives as possible p38 MAPK inhibitors. From the data reported here, it was observed that compound 26 was found to be the effective ligand which displayed better binding affinities (>200 $\mathrm{kcal} / \mathrm{mol}$ ) against all p38 MAPK isoforms when compared to original co-crystallized ligands $(149-191 \mathrm{kcal} / \mathrm{mol})$. Whereas inhibitors SB203580, SB202190, PD169316 resulted in dock scores between 164-189 kcal/mol. Therefore, it can be stated from the analysis that bipyrazole systems can be regarded as new class of p38 MAPK inhibitors and further experimental analysis would reveal possible features responsible for their activity.

\section{REFERENCES}

[1] W. Xia, M.T. Longaker, G.P. Yang. P38 MAP kinase mediates transforming growth factor-beta2 transcription in human keloid fibroblasts. Am J Physiol Regul Integr Comp Physiol 290 (2006) R501-8.

[2] M. Schiller, M. Böhm, S. Dennler, J.M. Ehrchen, A. Mauviel. Mitogen- and stress-activated protein kinase 1 is critical for interleukin-1-induced, CREB-mediated, cfos gene expression in keratinocytes Oncogene 25 (2006) 4449-57.

[3] A. Cuadrado and A. R. Nebreda. Mechanisms and functions of p38 MAPK signalling, Biochemical Journal 429 (2010) no. 3, 403-417.

[4] A. Cuenda and S. Rousseau. p38 MAP-kinases pathway regulation, function and role in human diseases, Biochimica et Biophysica Acta 1773 (2007) no. 8, 13581375 .

[5] Kumar S, Boehm J, Lee JC. p38 MAP kinases: key signalling molecules as therapeutic targets for inflammatory diseases, Nat Rev Drug Discov 2 (2003) $717-726$.

[6] AS Dhillon, S Hagan, O Rath and W Kolch. MAP kinase signalling pathways in cancer. Oncogene 26 (2007) 3279-3290.

[7] N. Deepak, Dr. A. Yesu babu, Dr. K. Kiran Kumar, In silico pathway analysis of MAPKs and computational investigation of bipyrazole analogs as novel p38-alpha MAPK inhibitors, International Journal of Bioinformatics Research and Applications (Accepted, In Production), 2015.

[8] F. Bakr Abdel-Wahab and M. Kamal Dawood. Synthesis and applications of bipyrazole systems. ARKIVOC i (2012) 491-545.

[9] Samir Bondock, Abd El-Gaber Tarhoni and A. Ahmed Fadda. Utility of cyanoacetic acid hydrazide in heterocyclic synthesis. ARKIVOC ix (2006) 113-156.

[10] C. Dominguez, D.A. Powers, N. Tamayo. P38 MAP kinase inhibitors: many are made, but few are chosen. Curr Opin Drug Discov Devel 8 (2005) 421-30. 